\title{
Regulation of Macrophage-Derived Fibroblast Growth Factor Release by Arachidonate Metabolites
}

\author{
Sem H. Phan, Bridget M. McGarry, Kathryn M. Loeffler, and Steven L. Kunkel \\ Department of Pathology, The University of Michigan Medical School, Ann Arbor
}

\begin{abstract}
The macrophage is a source of many mediators with direct and indirect fibrogenic potential. In this study, release of macrophage-derived fibroblast growth factor (MDGF) activity by murine peritoneal macrophages is examined with regard to its regulation by arachidonate metabolites. Upon stimulation with $10 \mu \mathrm{g} / \mathrm{ml}$ lipopolysaccharide (LPS), realdent peritoneal macrophages from CBA/J mice released MDGF activity into modia rapidly, reaching maximal levels in approximately $1 \mathrm{~h}$. Lyeates of these stimulated cells also rovealed significantly increased cell-asecciated MDGF acthity, composing $45 \%$ of the total assayable activity. This acthity, as assayed by radloactive thymidine incorporation by primary cultures of rat lung fibroblasts, was separable from interieukin-1 (IL-1) activity by reverse phase high performance liquid chromatography (HPLC). Furthermore, purffied murine IL-1 had no MDGF activity in this aseay system. This stimulated MDGF release was enhanced by the cylooxygenase inhibltors indomethacin, ibuprofen, and aspirin at micromolar concentrations, but inhiblted in a dose-dependent manner by prostaglandin $E_{2}\left(P G E_{2}\right)$. On the other hand, nordihydroguaiaretic acid (NDGA), a lipoxygenase inhibitor was inhibitory at 0.1 and $0.4 \mu \mathrm{M}$ but not at $2.5 \mu \mathrm{M}$. Zymosan-stimulated macrophages also markedly increased MDGF release, albelt with a different time course which was characterized by a delay of approximately $7 \mathrm{~h}$ before peak lovels were attained. Such stimulation, which is known to cause increased lipoxygenase activity, was also inhibited by $0.5 \mu M$ NDGA. In contrast, the lipoxygenase pathway products leukotrienes $B_{4}\left(L T B_{4}\right)$ and $C_{4}\left(L_{T} C_{4}\right)$ stimulated MDGF release in a dose-dependent $\left(10^{-10}-10^{-8} \mathrm{M}\right)$ manner, with $\mathrm{LTC}_{4}$ being more potent on a per unit dose basis. Stimulation by LTC $_{4}$ was inhibited by the putative leukotrione receptor antagonist, FPL55712, while LTD $_{4}$ and LTE $_{4}$ did not stimulate MDGF release, thus suggesting the mediation of this effect by specific LTC 4 receptors. These data suggest also that products of the cyclooxygenase and lipoxygenase pathways are potentially important both as exogenous (ie, derived from cells other than the macrophage itseli) and auto- or self-regulators of macrophage MDGF release. This, in tum, implles that cyclooxygenase products are antifibrogenic and important in maintaining or returning to the quibecent or normal state, whereas the lipoxygenase products are profibrogenic and important in induction of fibrosis or wound-healing and tissue repair. Any alteration in the balance between these two pathways may result in either a desirable or a harmful outcome.
\end{abstract}

Key words: macrophage, fibroblast growth factor

\section{INTRODUCTION}

There is mounting evidence that the macrophage is an important participant in the fibrogenic response. One of the earliest hints comes from a study showing inhibition of wound healing and scar formation in animals selectively depleted of macrophages [18]. Since this study, there have been multiple reports of macrophages as sources of macrophage-derived fibroblast growth factor (MDGF) and fibroblast collagen synthesis stimulatory and fibroblast chemotactic activities [19,20,29,30,32]. Additionally, the macrophage is a major source of certain arachidonate metabolites $[4,17,26]$, which have wellknown fibrogenic modulating activities $[1,8,10,16,20]$. These metabolites, both endogenously and exogenously (ie, from other cellular sources) derived, have been reported also to modulate the production or release of certain mediators by macrophages, such as interleukin 1 (IL-1) $[6,7,17,25]$. One of these metabolites, prostaglandin $E_{2}\left(P_{2} E_{2}\right)$, inhibits IL-1 release by lipopolysaccharide (LPS)-stimulated macrophages and may represent an important endogenous or autoregulator of IL-1 release [17].

Received August 10, 1986; accepted February 3, 1987.

Reprint requests: Sem H. Phan, Department of Pathology/M0602, The University of Michigan Medical School, Ann Arbor, MI 481090602 . 
Such modulation of macrophage mediator production will ultimately have an impact on the fibrogenic response, since many of these mediators, including the arachidonate metabolites themselves, have direct effects on fibroblast growth, movement, and protein metabolism $[1,8,10,16,19,20,29,30,32]$. The modulation of MDGF release by arachidonate metabolities has not been specifically studied $[24,31]$, although modulation of IL-1 or IL-1-like molecules with putative MDGF activity has been reported $[6,17]$. In preliminary studies, we have reported that the cyclooxygenase inhibitor indomethacin enhanced MDGF secretion by LPS-stimulated peritoneal macrophages, while $\mathrm{PGE}_{2}$ was inhibitory [17]. In the present study, we have extended these studies and also examined the kinetics of MDGF release, the effects of other cyclooxygenase and lipoxygenase inhibitors, and leukotrienes on such stimulated release. Additionally, data were presented showing that the measured MDGF activity was distinct from IL-1.

\section{MATERIALS AND METHODS \\ Reagents}

LPS (from Escherichia coli, serotype 0111:B4), nordihydroguaiaretic acid (NDGA), indomethacin, aspirin (acetylsalicylic acid), and acetaminophen were purchased from Sigma Chemical Co. (St. Louis, MO). RPMI-1640, Dulbecco's modified Eagle's medium (DMEM), penicillin-streptomycin-fungizone stock solution, fetal calf serum (FCS), and L-glutamine were obtained from GIBCO (Grand Island, NY). Trypsin (1:250) for isolation and passaging cells was from Difco Laboratories (Detroit, MI). Ibuprofen was obtained from the Upjohn Co. (Kalamazoo, MI). BW755c was from Wellcome Research Laboratories (Beckenham, England). $\mathrm{PGE}_{2}$ was a gift from Pfizer Central Research (Groton, CT). Dr. J. Rokach (Merck Frosst Laboratories, Quebec, Canada) kindly provided the $\mathrm{LTB}_{4}$ and $\mathrm{LTC}_{4}$. [Methyl$\left.{ }^{3} \mathrm{H}\right]$ thymidine $(6.7 \mathrm{ci} / \mathrm{mmol})$ was purchased from New England Nuclear (Boston, MA). All other reagents were of analytical reagent grade or better.

\section{Cells}

Resident mouse peritoneal macrophages were obtained by lavaging the peritoneal cavities of female CBA/J mice (The Jackson Laboratories, Bar Harbor, ME) with sterile RPMI exactly as previously described [17]. Adherent cells were then incubated in serum-free RPMI containing $10 \mu \mathrm{g} / \mathrm{ml}$ LPS with or without various arachidonate metabolities or inhibitors of cyclooxygenase or lipoxygenase pathways [17]. Except for the time course studies, the incubation continued for 16-18 h (overnight). Conditioned media were then harvested, and the volume was determined. After counting of the adherent cells, the media were then adjusted to the equivalent of $10^{5}$ adherent cells/ml prior to filter sterilization and storage at $-20^{\circ} \mathrm{C}$ until ready for mediator assay. When zymosan was used, the media were centrifuged to remove the zymosan prior to filter sterilization. Where indicated, macrophage lysates were prepared by repeated freezing and thawing of the adherent cells after collection of conditioned media, washing and resuspension in fresh RPMI [17]. The supernatant after removal of cellular debris by centrifugation was filter sterilized and stored frozen until assay, and referred to as the cell-associated fraction or lysate.

Rat lung fibroblasts were isolated from specific pathogen-free male Fisher 344 rats (Charles River, Portage, MI) by trypsinization of fresh lung mince as previously described [22]. Cells were maintained in DMEM containing 10\% FCS. Only cells from passages 3-15 were used for MDGF assay [17,22]. In control experiments, these rat lung fibroblasts did not differ significantly from murine lung fibroblasts in their responsiveness to macrophage-conditioned media (data not shown). Since the rat cells were easier to maintain in culture, all experiments were undertaken with these cells.

Macrophage-conditioned media and lysates at $10 \%$ final concentrations were assayed for MDGF activity as previously described [17]. Data were expressed as cpm incorporated per microtiter well. Quiescent untreated control fibroblasts usually incorporated $200-700 \mathrm{cpm} /$ well while the positive controls (in the presence of $10 \%$ FCS) usually incorporated 7,000-10,000 cpm/well.

\section{Chromatography}

Conditioned media from LPS-stimulated macrophages were concentrated tenfold prior to analysis by reverse phase high performance liquid chromatography (HPLC). The instrumentation consisted of a Varian 5560 chromatograph equipped with a UV-200 UV-visible variable wavelength detector and a model $\mathbf{4 0 2}$ data system (Varian Instruments, Palo Alto, CA). A Supelco (Bellefonte, PA) LC-304 column was used for this analysis. This column $(4.6 \mathrm{~mm} \times 15 \mathrm{~cm})$ contained $30-\mu \mathrm{m}$-diameter pore size resin with a $\mathrm{C}_{4}$ bonded phase. The column was eluted with a linear gradient of $10-50 \%$ B over 40 minutes, with solvent $\mathrm{A}$ composed of $0.05 \mathrm{M}$ ammonium acetate (pH 6.8), in $\mathrm{H}_{2} \mathrm{O}$ and solvent $\mathrm{B}$ composed of the same buffer composition, but in $50 \% \mathrm{CH}_{3} \mathrm{CN}$ (in $\mathrm{H}_{2} \mathrm{O}$ ). The column temperature was maintained at $35^{\circ} \mathrm{C}$, and the flow rate was $1 \mathrm{ml} / \mathrm{min}$. The effluent was detected at 230 $\mathrm{nm}$ and $1 \mathrm{ml}$ fractions were collected, dried by vacuum centrifugation (Speed Vac, Savant Instruments, Hicksville, NY), and subsequently assayed for MDGF and IL1 activities after resuspension in RPMI. 


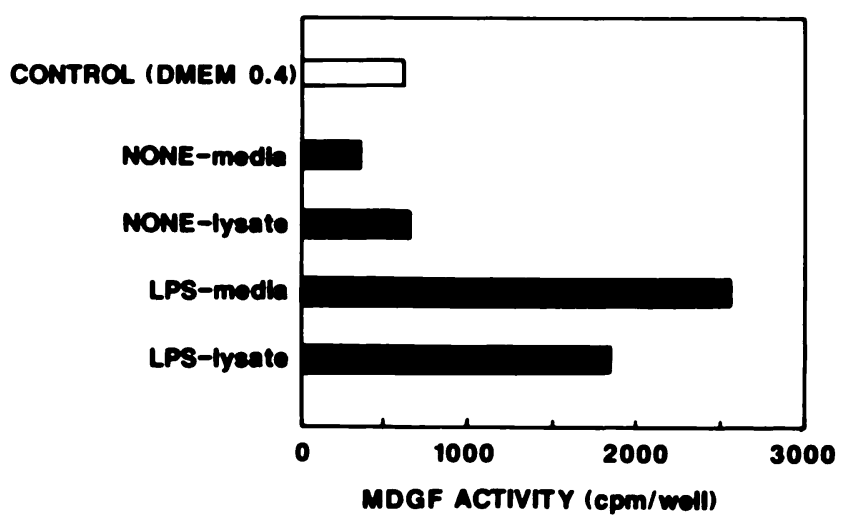

Fig. 1. Stimulation of MDGF activity by LPS. Redident murine peritoneal macrophages were incubated for $16 \mathrm{~h}$ in the pres. ence (LPS) or abeence (NONE) of $10 \mu \mathrm{g} / \mathrm{ml}$ LPS. Conditioned media were then eeparated from the adherent cell layer, and teated for MDGF acthvity as described in Materials and Mothods. The cell layer was frozen and thawed to lyee the celle, and the supernate obtained after centrffugation was also asaayed for MDGF activity (lyante fraction). Control rofers to cpm incorporated in the presence of DMEM containing 0.4\% FCS. Data expreased as the means of triplicate determinations.

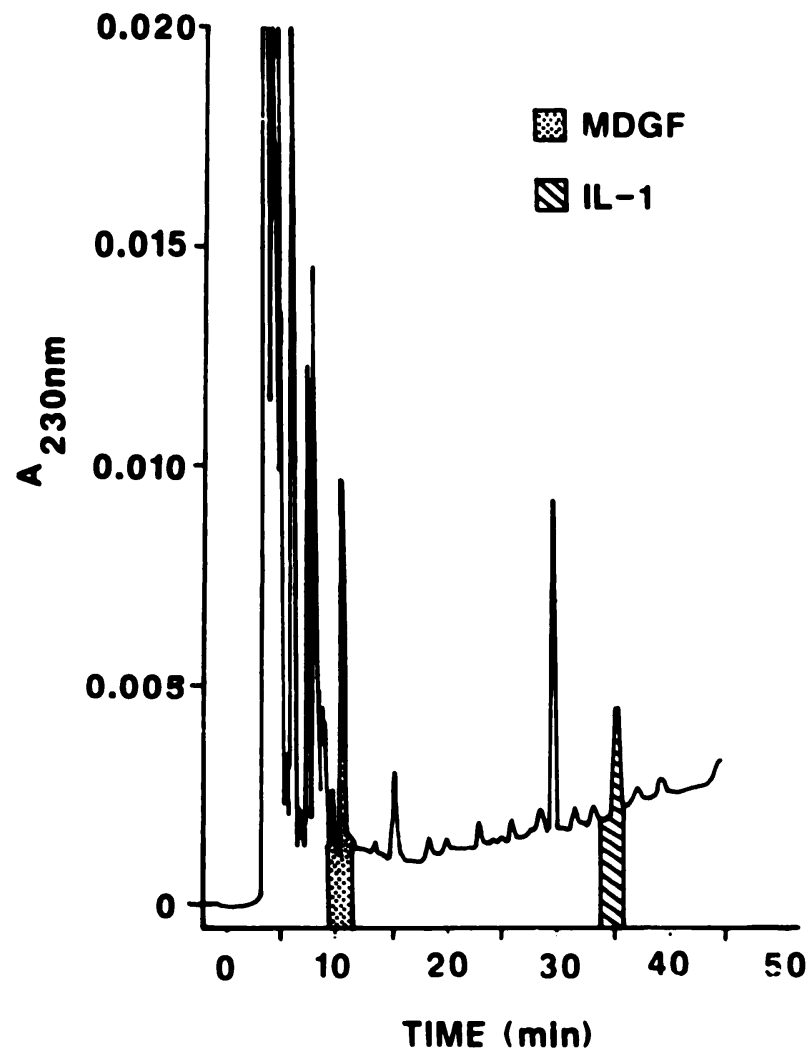

Fig. 2. Roverse phase HPLC eeparation of MDGF from IL-1. LPS-stimulated macrophage conditioned medla were concen trated tenfold, and injected onto a $C_{4}$ reveree phase column (eec Materials and Mothods for details). The efmuent was monHored at $230 \mathrm{~nm}$ and 1- $\mathrm{ml}$ fractions wore collected, dried, and aseayed for MDGF and IL-1 activities.

\section{Interleukin-1 Assay}

This was done exactly as described previously [17], using a modification of the method of Mizel et al [21].

\section{Statistical Analysis}

Student's t-test was used for comparing means of experimental versus control groups, with $p<0.05$ being statistically significant.

\section{RESULTS}

LPS is a potent stimulator of macrophage mediator release This effect was also observed in terms of MDGF activity: $10 \mu \mathrm{g} / \mathrm{ml}$ of LPS caused a 2-5-fold increase in the MDGF activity of macrophage-conditioned media when compared to control media of unstimulated cells. A typical result is shown in Figure 1, wherein an approximate fivefold stimulation was seen. This stimulated activity was also found in the lysates of LPS-stimulated macrophages, although the degree of stimulation was only slightly greater than twofold that of the lysates from unstimulated cells (Fig. 1). In terms of activity, the lysate contained approximately $45 \%$ of the total assayable activity (lysate plus media). Addition of the same dose of LPS directly into the MDGF assay failed to affect fibroblast thymidine incorporation (data not shown).

Since LPS-stimulated macrophages also release IL-1 $[13,17,21]$, and IL-1 has been reported to have FGF-like activity [27], the question arose as to whether this MDGF activity could be due to IL-1. In an attempt to clarify this issue, concentrated conditioned media from LPS-stimulated macrophages were fractionated by reverse phase HPLC. The resulting chromatogram showed that MDGF activity with a retention time centered around $10 \mathrm{~min}$ was clearly separable from fractions exhibiting IL-1 activity with a retention time of approximately $19 \mathrm{~min}$ (Fig. 2). The IL-1-containing fractions showed no detectable MDGF activity, except in the presence of high concentrations of serum ( $>5 \%$ ), while the MDGF positive peak was inactive in the IL-1 assay. Thus, this LPS-induced MDGF activity was physically distinct from IL-1, as have also been shown by others $[3,9,11]$. Finally, Table 1 shows the inability of purified murine IL-1 (a gift of Dr. M. Kluger, Department of Physiology, University of Michigan) [21] to significantly stimulate thymidine incorporation by fibroblasts in the MDGF assay.

The time course of this LPS stimulation of MDGF release was rapid, as shown in Figure 3. Maximal MDGF activity in conditioned media was observed within the first hour after addition of LPS. Simultaneous addition of the cyclooxygenase inhibitor indomethacin with the LPS caused an approximately 50\% increase in MDGF activity release above that of LPS alone (Fig. 3). To examine this phenomenon further, the dose dependence of this effect 
TABLE 1. Effect of Murine IL-1 on Fibroblasts

\begin{tabular}{llr}
\hline Treatment & \multicolumn{1}{c}{ Dose } & MDFG activity \\
\hline None & $10 \%$ & $398 \pm 42$ \\
MDGF $^{\text {b }}$ & - & $2,672 \pm 97$ \\
IL-1 $^{c}$ & 0.01 units $/ \mathrm{ml}$ & $439 \pm 69$ \\
& 0.1 units/ml & $316 \pm 47$ \\
& 1.0 units $/ \mathrm{ml}$ & $288 \pm 51$ \\
\hline
\end{tabular}

activity assayed as described in Materials and Methods, and expressed as mean cpm/ well $\pm S E(N=6)$.

bThis represents conditioned media of LPS-stimulated macrophages.

'This represents purified murine IL-1 [21].

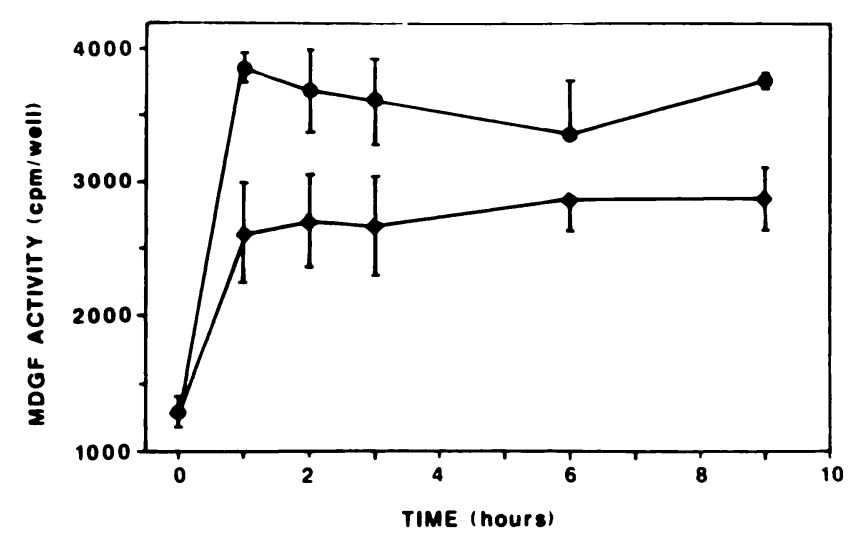

Fig. 3. Kinotics of LPS-Induced MDGF release. Macrophages were incubated in the presence of $10 \mu \mathrm{g} / \mathrm{ml}$ LPS, with $(O-0)$ or without $(\diamond-\uparrow) 1 \mu \mathrm{M}$ indomethacin. At the Indicated times, after addition of LPS, conditioned medla were collected and asaayed for MDCF activity. Data presented as mean $\pm S E, N=6$.

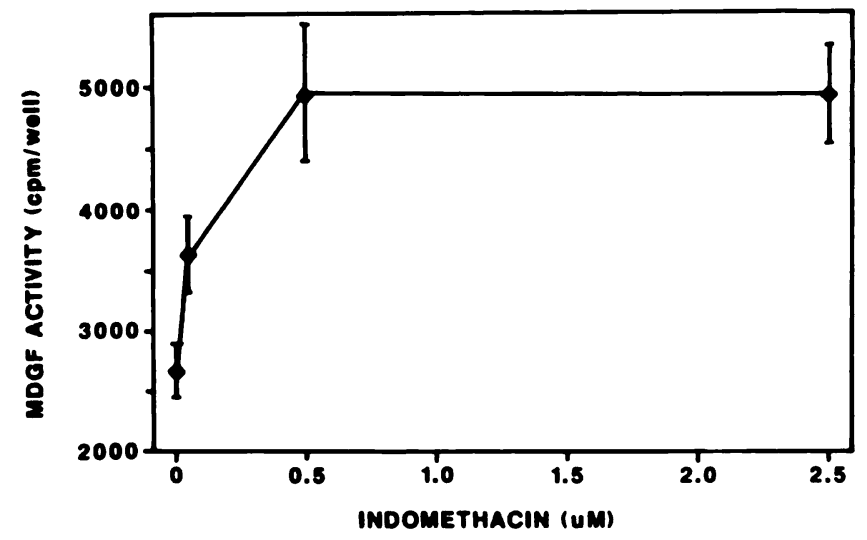

Fig. 4. Dose-response curve for indomethacin. Indomothacin were added at the indicated doses to LPS $(10 \mu \mathrm{g} / \mathrm{ml})$ stimulated macrophages. After $16 \mathrm{~h}$, the conditioned modia were collected and aseayed for MDGF activity. Data expreseed as mean $\pm S E$, $\mathbf{N}=\mathbf{6}$.

was examined along with the effects of other arachidonate metabolic inhibitors.

\section{The Role of Cyclooxygenase Metabolites}

The stimulatory effects of indomethacin were dosedependent, saturating at doses greater than $5 \times 10^{-7} \mathrm{M}$
(Fig. 4). Addition of these doses of indomethacin directly into the MDGF assay or into macrophage-conditioned media did not affect significantly the rate of thymidine incorporation by the target fibroblasts (Table 2). Thus, any effect that was observed was due to the effect of indomethacin on MDGF production and/or release. In order to examine if this stimulatory effect was shared by other cyclooxygenase inhibitors, several of them were tested for their effects on LPS-induced MDGF release. Figure 5A shows that this property was shared by ibuprofen and aspirin, although the effects were smaller on a per unit dose basis. Acetaminophen also stimulated MDGF release, although it is not known as a cyclooxygenase inhibitor [17]. On the other hand, piroxicam and the lipoxygenase inhibitor BW755c were ineffective in influencing LPS-induced MDGF release. These results do not seem to correlate with the abilities of these agents to inhibit $\mathrm{PGE}_{2}$ production as previously reported [17], although Figure 5B confirms the dose-dependent inhibitory effect of $\mathrm{PGE}_{2}$ on LPS-induced MDGF release [17]. $\mathrm{PGE}_{2}$ at these dilutions $\left(\leqslant 1 \times 10^{-7}\right)$ did not significantly inhibit the fibroblasts in the MDGF assay (Table 2). The data would thus suggest the possibility that these agents may exert their effect via multiple mechanisms in addition to the inhibition of the cyclooxygenase pathway, unlike the situation with IL-1 release, wherein stimulation of its release by these inhibitors correlated with the inhibition of macrophage $\mathrm{PGE}_{2}$ production [17].

\section{The Role of Lipoxygenase Metabolites}

In contrast to the stimulatory effects of the cyclooxygenase inhibitors, the lipoxygenase inhibitor NDGA inhibited LPS-induced MDGF release in a dose-dependent manner (Fig. 6). The inhibitory effect, however, was not seen at $2.5 \mu \mathrm{M}$, the highest dose tested. This was possibly due to concomitant cyclooxygenase inhibition by high doses of NDGA, as has been previously reported [5]. The inhibitory effect of lipoxygenase inhibition on MDGF release was also suggested by the BW755c data (Fig. 5A), showing slight inhibition of MDGF release by this inhibitor of $\mathrm{LTC}_{4}$ production [5]. NDGA added directly to the fibroblasts did not affect thymidine incorporation significantly (Table 2). 

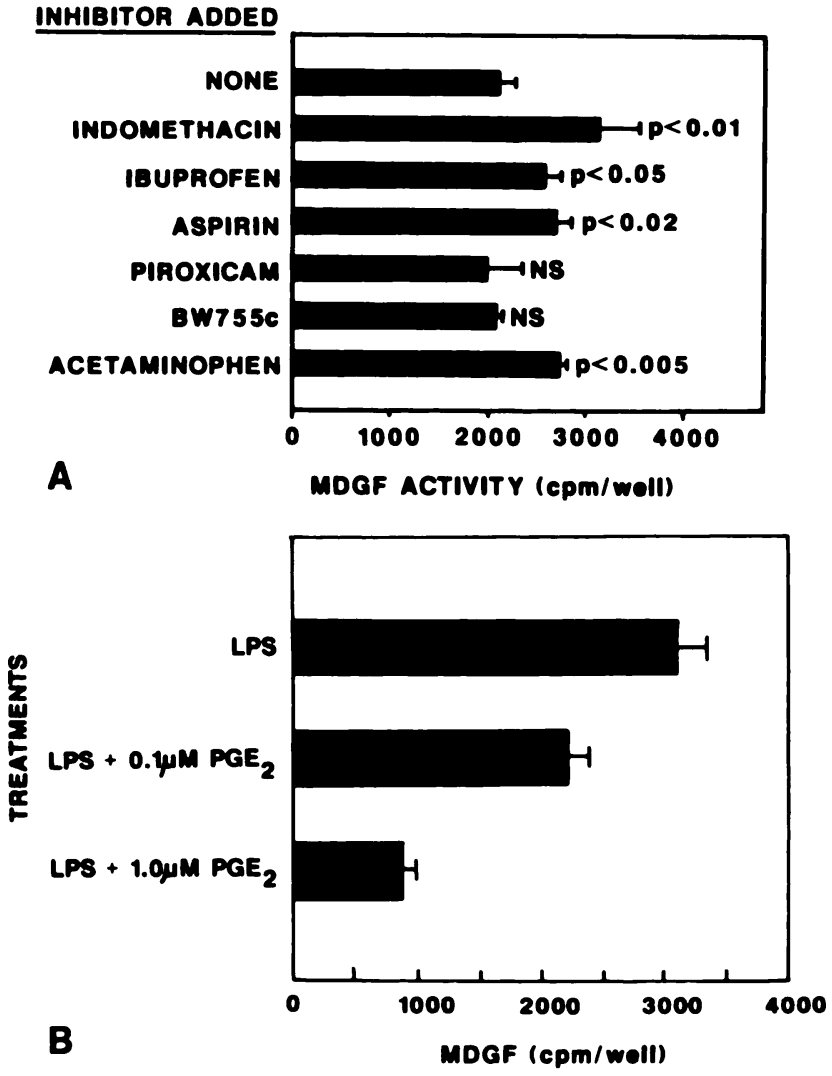

Fig. 5. Effects of cyclooxygenase Inhibitore and PGE. A: Inhibltors $(1 \mu \mathrm{M})$ were added to LPS $(10 \mu \mathrm{g} / \mathrm{ml})$ stimulated macrophage cultures. After 16 h conditioned media were collected and assayed for MDGF. Data wore presented as moans \pm SE $(N=6$, except for indomothacin, where $N=5$ ). P value indlcated the results of comparing their mean values to the LPS (no inhibitor) control mean value, by Student's t-teet. NS refers to $p \geqq 0.05$. B: $P G E_{2}$ was added to LPS-stimulated macrophages at the indlicated concentrations, and the conditioned medla wore harvested to assay for MDGF activity. Data represent means \pm SE $(N=6)$.
Since LPS does not cause significant stimulation of the production of lipoxygenase metabolites [14,17], this NDGA effect was further examined using zymosan, an agent known to cause stimulation of the lipoxygenase pathway $[14,17]$. Addition of zymosan also caused increased production of MDGF activity, but with a different kinetics compared to LPS. Instead of almost instantaneous release as seen with LPS (Fig. 3), a lag phase of approximately $6 \mathrm{~h}$ was observed with zymosan stimulation before maximal release was attained at time points greater than $9 \mathrm{~h}$ (Fig. 7). The inhibitory effects of NDGA $\left(5 \times 10^{-7} \mathrm{M}\right)$ was again observed, thus suggesting that inhibition was occurring at a step or steps common to macrophage activation by these two different agents. In view of the antilipoxygenase activity of NDGA, several lipoxygenase pathway products were tested for their ability to modulate, especially stimulate, MDGF release by macrophages.

Addition of $\mathrm{LTB}_{4}$ and $\mathrm{LTC}_{4}$ to macrophage cultures, caused a dose-dependent increase in MDGF release (Fig. 8). $\mathrm{LTC}_{4}$ stimulation was significantly greater than $\mathrm{LTB}_{4}$ stimulation on a per unit dose basis, although their halfmaximal effective doses $\left(E D^{50}\right)$ were similar at approximately $5 \times 10^{-9} \mathrm{M}$. Addition of these leukotrienes directly to fibroblast cultures at these dilutions had no significant effect on thymidine incorporation (data not shown). These effects were thus the result of actual increase in MDGF release by the macrophages. The stimulatory effect of $\mathrm{LTC}_{4}$ was blocked by the putative sulfidopeptide leukotriene receptor antagonist FPL55712, suggesting that the effect was mediated by an LTC $_{4}$ receptor on the macrophage. Although FPL55712 has greater specificity for $\mathrm{LTD}_{4}$, at least in guinea pig trachea [28], this leukotriene as well as $\mathrm{LTE}_{4}$ did not stimulate MDGF release at comparable doses (data not shown).

TABLE 2. Effects of Indomethacin, NDGA, and PGE, on MDGF Aseay

\begin{tabular}{|c|c|c|}
\hline Treatment & Dose & {$\left[{ }^{3} \mathrm{H}\right]$ Thymidine incorporation } \\
\hline None & - & $346 \pm 69$ \\
\hline MDGF $^{b}$ & $10 \%$ & $2,100 \pm 193$ \\
\hline Indomethacin ${ }^{c}$ & $1 \times 10^{-7} \mathrm{M}$ & $511 \pm 103$ \\
\hline Indomethacin & $2.5 \times 10^{-7} \mathrm{M}$ & $623 \pm 122$ \\
\hline MDGF + Indomethacin ${ }^{d}$ & $10 \%$ & $2,351 \pm 271$ \\
\hline NDGA $^{c}$ & $1 \times 10^{-7} \mathrm{M}$ & $392 \pm 101$ \\
\hline NDGA & $2.5 \times 10^{-7} \mathrm{M}$ & $569 \pm 92$ \\
\hline MDGF + NDGA ${ }^{d}$ & $10 \%$ & $2,009 \pm 215$ \\
\hline $\mathrm{PGE}_{2}$ & $10^{-7} \mathrm{M}$ & $403 \pm 103$ \\
\hline $\mathrm{MDFG}+\mathrm{PGE}_{2}$ & $10 \%$ & $1,603 \pm 100$ \\
\hline
\end{tabular}

"MDGF assay was done as described in Materials and Methods, and results are expressed as mean cpm/ well $\pm \mathrm{SE}(\mathrm{N}=3)$.

bThis represents LPS-stimulated macrophage conditioned media.

cAdded directly to the fibroblasts, and incubated as for the standard MDGF assay, but without addition of conditioned media.

This represents macrophage conditional media as in footnote $b$ above, into which $10^{-6} \mathrm{M}$ indomethacin, NDGA, or $\mathrm{PGE}_{2}$ had been added just prior to the MDGF assay, resulting in a final concentration of $10^{-7} \mathrm{M}$ in the MDGF assay for each of these compounds. 


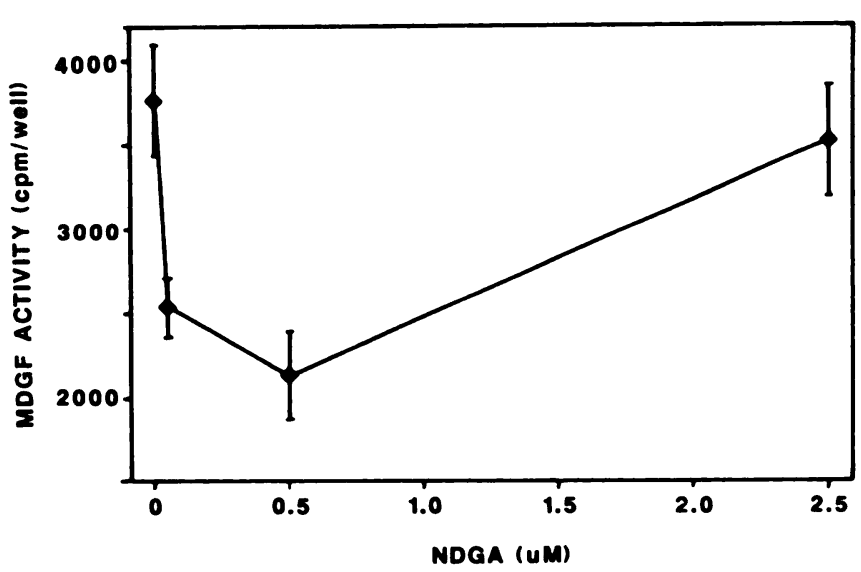

Fig. 6. Dose-response curve for NDGA. The experimental protocol was identical with that described in the legend to Figure 4, except NDGA at the indicated doses was added, instead of indomothacin. Data represent means $\pm \operatorname{SE}(N=6)$.

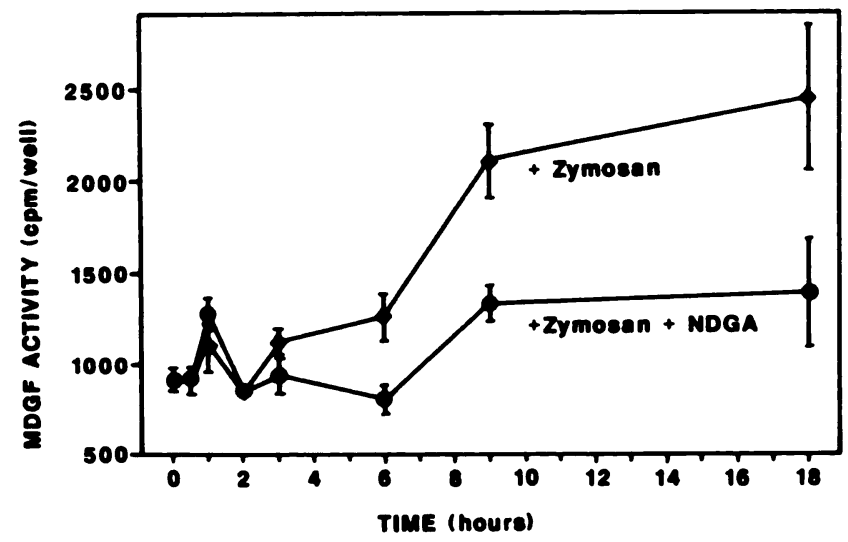

Fig. 7. Kinetics of zymosan-Induced MDGF release. Experimontal protocol was identical with that described in legend to Figure 3, except zymosan $(100 \mu \mathrm{g} / \mathrm{ml})$, with or without $1 \mu \mathrm{M}$ NDGA, was added at time zero, Instead of LPS. Data represent means $\pm S E, N=6$.

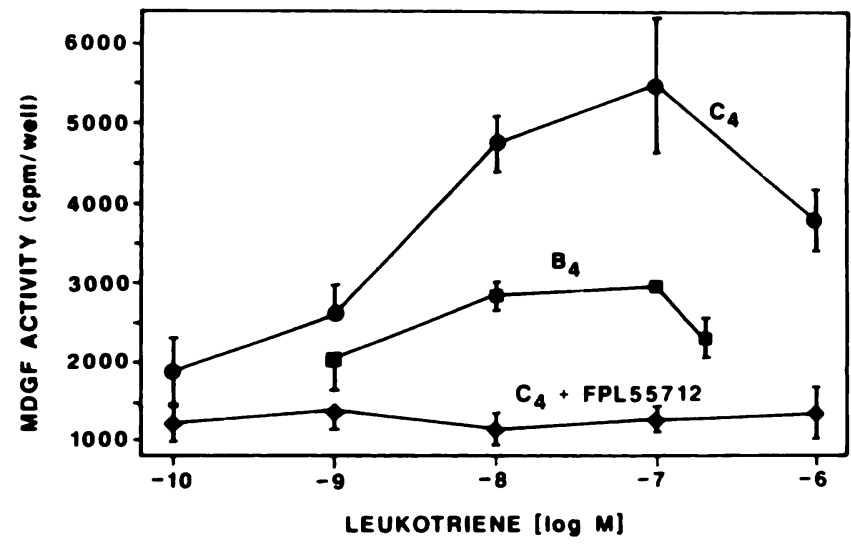

Fig. 8. Effects of leukotrienes on MDGF release. Macrophages were incubated with the indicated concentrations of leukotriene $\left(C_{4}=L_{T} C_{4}, B_{4}=L_{T} B_{4}\right)$ with or without $5 \mu M$ FPL55712 added 15 min prior to LTC $_{4}$ addition, for $16 \mathrm{~h}$. Media were then assayed for MDGF activity. Data represent moans \pm SE $(\mathbf{N}=6)$.
These data complemented the NDGA data and confirmed the possibility that lipoxygenase pathway products, either endogenously produced by the macrophage, or exogenously introduced, are stimulatory to MDGF release by the macrophage.

\section{DISCUSSION}

Arachidonate metabolites appear to have complex bidirectional effects on macrophage mediator production $[6,7,17,24,25,31]$. This state of affairs appear to extend also to MDGF release. LPS caused a rapid increase in MDGF release which was further stimulated in a dosedependent manner by the cyclooxygenase inhibitor indomethacin. This secreted MDGF activity was physically distinct from IL-I, as indicated by the ability of reverse phase HPLC to separate these two activities. That this MDGF was different from IL-1 was also supported by the inability of purified murine IL-1 to significantly stimulate thymidine incorporation by fibroblasts used in the MDGF assay. Further support was also provided by studies from other laboratories $[3,9,11]$, and by the difference in the effectiveness of various cyclooxygenase inhibitors to modulate IL-1 [17] versus MDGF release as discussed below.

The stimulatory property of indomethacin was also shared by ibuprofen and aspirin, but not by piroxicam and BW755c. In previous studies, we have shown that indomethacin, ibuprofen, and piroxicam inhibited $\mathrm{PGE}_{2}$ production by these macrophages [17], while asprin was inhibitory at much higher doses [17]. If the mechanism of the stimulation is due to decreased production of $\mathrm{PGE}_{2}$, then all three should stimulate MDGF release. But the efficacy of aspirin and the inability of piroxicam to stimulate MDGF release contrasted sharply with their ability or lack of ability to inhibit $\mathrm{PGE}_{2}$ production. This would suggest additional (other than by cyclooxygenase inhibition) mechanism(s) by which indomethacin, aspirin, and ibuprofen could stimulate MDGF release. But it remained clear that at least a product of the cyclooxygenase pathway, namely $P G E_{2}$, was inhibitory to LPS-induced MDGF release, and this represented one mechanism by which cyclooxygenase inhibition could result in stimulation (or release from inhibition) of LPS-induced MDGF release. The ineffectiveness of BW755c with respect to LPS-induced MDGF release may be due to its ability to also inhibit the lipoxygenase pathway [5], thus simultaneously inhibiting a pathway whose products could directly stimulate MDGF release, as shown by the leukotriene data. These complex dose-dependent effects are partly the result of the lack of specificity of these inhibitors at relatively high doses [5]. The stimulatory effect of acetaminophen is unexpected, and is in direct contrast to its lack of effect on LPS-induced IL-1 release [17], which provides further support for the conclusion 
that MDGF is distinct from IL-1. The mechanism of this stimulation of LPS-induced MDGF release by acetaminophen remains to be elucidated.

In contrast to cyclooxygenase inhibition, the lipoxygenase inhibitor NDGA inhibited in a dose-dependent manner the stimulation of MDGF release by both LPS and zymosan. The latter stimulus caused MDGF release only after a 6-7-h lag phase, and was associated also with stimulation of the lipoxygenase pathway [14], in contrast to LPS, which caused primarily the stimulation of the cyclooxygenase pathway $[14,17]$. Despite these differing mechanisms of stimulation by these two dissimilar substances, the ability of NDGA to inhibit MDGF release would suggest the existence of a common pathway in macrophage activation and MDGF release which would require a product or products of the lipoxygenase pathway. This possibility was strengtheneed by the ability of $\mathrm{LTB}_{4}$ and $\mathrm{LTC}_{4}$ to stimulate MDGF release directly, and by the indication that at least the $\mathrm{LTC}_{4}$ effect may be mediated by specific receptors on the macrophage. The lack of effect of $\mathrm{LTD}_{4}$ and $\mathrm{LTE}_{4}$ provided support for the specificity of such $\mathrm{LTC}_{4}$ receptors. The loss of inhibitory effect by NDGA at $2.5 \mu \mathrm{M}$ may be due to loss of its specificity at high doses, as suggested by its ability also to inhibit cyclooxygenase activity at high doses [5]. Such inhibition could result in decreased $\mathrm{PGE}_{2}$ production, thus negating the inhibitory effects of decreased leukotriene production.

An important conclusion from these data is the in vivo or physiologic significance of this modulation by arachidonate metabolites. This myriad of effects on MDGF release was seen at doses which were physiologically relevant $\left(<10^{-8} \mathrm{M}\right)$ and likely to be encountered at sites of tissue injury and inflammation. The sources of these metabolites are both endogenous (ie, the macrophage itself) and exogenous (eg, polymorphonuclear leukocytes, platelets, endothelial cells, etc). Whether the end result leads to increase fibroblast proliferation and fibrosis, or to inhibition of scar formation, would partly depend on the resulting balance between the opposing effects of cyclooxygenase versus lipoxygenase product accumulation. These opposing effects of cyclooxygenase versus lipoxygenase metabolites on MDGF release are similar to effects seen with regard to other macrophage mediators, such as IL-1 [6,7,17,24,25,31]. There are, however, clear differences between mediators under certain conditions of cyclooxygenase inhibition for instance. As noted above, despite inhibition of $\mathrm{PGE}_{2}$ production by piroxicam, MDGF release was not affected, while IL1 release was increased significantly [17]. The mechanism behind this discrepancy is unknown and, doubtless, complex. This complexity is partly attributable to the complex events associated in phagocytic cell activation. Nevertheless, the potent effects of $\mathrm{PGE}_{2}$ and $\mathrm{LTC}_{4}$ in modulating MDGF release would suggest their in vivo importance as endogenous, as well as exogenous, inhibitor and stimulator, respectively, of fibroblast proliferation. Since different stimuli result in different spectra of arachidonate metabolite released, the ultimate effect on MDGF release will depend on the net effect of these different metabolites, both inhibitory and stimulatory. It would, therefore, be impossible to predict an effect merely on the basis of quantitation of products of one pathway. For example, LPS-stimulation is known to cause increased $\mathrm{PGE}_{2}[14,17]$ and MDGF release, but not or negligible $\mathrm{LTC}_{4}$ release [14,17]. On the other hand, zymosan causes increased release of all three mediators ( $\mathrm{PGE}_{2}, \mathrm{MDGF}$, and $\mathrm{LTC}_{4}$ ) [14], albeit with different kinetics. On the basis of the lipoxygenase pathway profile, one would have predicted, incorrectly, that zymosan would be a more potent stimulator of MDGF release, while LPS would be inhibitory.

The mechanism of this regulation by arachidonate metabolites remains to be elucidated. This is likely to occur at three major possible sites. First, such regulation could occur at the level of receptor abundance or expression, and many of the stimuli for phagocytic cell activation are receptor mediated [15]. Second, arachidonate metabolites have potent effects on intracellular calcium concentrations [33], as well as other intracellular messengers associated with cell activation [12]. These are bound to impact on MDGF and other mediator release. Finally, there is evidence that LPS stimulation results in increased transcription of certain polypeptide mediator genes, such as tumor necrosis factor [2]. However, similar studies on how arachidonate metabolites would regulate MDGF gene transcription must await the availability of specific cDNA probes for this mediator.

The susceptibility of MDGF release to inhibition by lipoxygenase inhibitors and $\mathrm{PGE}_{2}$ suggests new strategies for the development of therapeutic agents to combat undesirable fibrosis, such as in hepatic cirrhosis and interstitial pulmonary fibrosis. The possible efficacy of such agents is suggested by the ability of lipoxygenase inhibitors to inhibit bleomycin-induced pulmonary fibrosis [23]. This effect of NDGA is associated with the inhibition of alveolar macrophage-derived MDGF release [23]. Thus, the development of more specific lipoxygenase inhibitors with less undesirable side effects may be useful for controlling fibrotic diseases.

\section{ACKNOWLEDGMENTS}

This work is supported in part by grants from the National Institutes of Health (HL28737, HL31963, and HL31237) and the American Heart Association and its Michigan Affiliate. Drs. Phan and Kunkel are Established Investigators of the American Heart Association. 
We would like also to acknowledge the excellent editorial assistance by Kathleen Atkins.

\section{REFERENCES}

1. Baum, B.J., Moss, J., Breul, S.D., and Crystal, R.G. Association in normal human fibroblasts of elevated levels of adenosine $3^{1} S^{1}$-monophosphate with a selective decrease in collagen production. J. Biol. Chem., 253, 3391, 1978.

2. Beutler, B., Krochin, N., Milsark, I.W., Leudke, C., and Cerami, A. Control of cachectin (tumor necrosis factor) synthesis: Mechanisms of endotoxin resistance. Science 232,977, 1986.

3. Bitterman, P.B., Rennard, S.I. Hunninghake, G.W., and Crystal, R.G. Human alveolar marcophage growth factor for fibroblasts: Regulation and partial characterization. J. Clin. Invest. 70,808, 1982.

4. Bonney, R.J., Opas, E.E., and Humes, J.L. Lipoxygenase pathways of macrophages. Fed. Proc. 44, 2933, 1985.

5. Bonney, R.J., and Humes, J.L. Physiological and pharmacological regulation of prostaglandin and leukotriene production by macrophages. J. Leukocyte Biol. 35,1, 1984.

6. Dinarello, C.A., Bishai, I., Rosenwasser, L.J., and Coceani, F. The influence of lipoxygenase inhibitors on the in vitro production of human leukocyte pyrogen and lymphocyte activating factor (interleukin-1). Int. J. Immunopharmacol. 6,43, 1984.

7. Dinarello, C.A., Marney, S.O., and Rosenwasser, L.J. Role of arachidonate metabolism in the immunoregulatory functon of human leukocyte pyrogen/lymphocyte activating factor/interleukin-1. J. Immunol. 130,890, 1983.

8. Dodge, W., and Thomas, M. The effect of 5-hydroxyeicosatetraenoic acid on the proliferation of granulocyte progenitors and embryonic fibroblasts of the chick. Biophys. Res. Commun. $131,731,1985$.

9. Dohlman, J.G., Payan, D.G., and Goetzl, E.J. Generation of a unique fibroblast-activating factor by human monocytes. Immunology 52,577, 1984.

10. Elias, J.A., Zurier, R.B., Schreiber, A.D., Leff, J.A., and Daniele, R.P. Monocyte inhibition of lung fibroblast growth: Relationship to fibroblast prostaglandin production and density-defined monocyte subpopulation. J. Leukocyte Biol. 37,15, 1985.

11. Estes, J.E., Pledger, W.J., and Gillespie, G.Y. Macrophage derived-growth factor for fibroblasts and interluekin-1 are distinct entities. J. Leukocyte Biol. 35,115, 1984.

12. Fantone, J.C., Duque, R.E., and Phan, S.H. Prostaglandin modulation of $\mathrm{N}$-formylmethionylleucylphenylalanine-induced transmembrane potential changes in rat neutrophils. Biochim. Biophys. Acta 804,205, 1984.

13. Gery, I.P., Davies, J., Derr, N.K., and Barranger, J.A. Relationship between production and release of lymphocyte activating factor (IL-1) by murine macrophages. I. Effects of various agents. Cell. Immunol. 131,2965, 1981.

14. Humes, J.A., Sadowski, S., Galavage, M., Goldenberg, M.M., Subers, E., Bonney, R.J., and Kuehl, F.A., Jr. Evidence for two sources of arachidonic acid for oxidative metabolism by mouse peritoneal macrophages. J. Biol. Chem. 257,1591, 1982.

15. Johnston, R.B., Jr., and Kitagawa, S. Molecular basis for the enhanced respiratory burst of activated macrophages Fed. Proc. 44,2927, 1985.

16. Ko, S.D., Page, R.C., and Narayanan, A.S. Fibroblast hetero- geneity and prostaglandin regulation of subpopulations. Proc. Natl. Acad. Sci. U.S.A. 74,3429, 1977.

17. Kunkel, S.L., Chensue, S.W., and Phan, S.H. Prostaglandins as endogenous mediators of interleukin-1 production. J. Immunol. 136,186, 1986.

18. Leibovich, S.J., and Ross, R. The role of the macrophage in wound repair. A study with hydrocortisone and antimacrophage serum. Am. J. Pathol. 78, 71, 1975.

19. Leibovich, S.J., and Ross, R. A macrophage-dependent factor that stimulates the proliferation of fibroblasts in vitro. Am. J. Pathol. 84,501, 1976.

20. Mensing, H., and Czarnetzki, B.M. Leukotriene $B_{4}$ induces in vitro fibroblast chemotaxis. J. Invest. Dermatol. 82,9, 1984.

21. Mizel, S.B., Dukovich, M. and Rothstein, J. Preparation of goat antibodies against interleukinl: Use of an immunosorbent to purify interleukin 1. J. Immunol. 131,1834, 1984.

22. Phan, S.H., Varani, J., and Smith, D. Rat lung fibroblast collagen metabolism in bleomycin-induced pulmonary fibrosis. J. Clin. Invest. 76, 241, 1985.

23. Phan, S.H., and Kunkel, S.L. Inhibition of bleomycin-induced pulmonary fibrosis by nordihydroguiaretic acid: The role of alveolar macrophage activation and mediator production. Am. J. Pathol. 124, 343, 1986.

24. Polla, B., de Rochemontriex, B., Junod, A.F., and Dayer, J.-M. Effects of $\mathrm{LTB}_{4}$ and $\mathrm{Ca}^{++}$ionophore $\mathrm{A} 23187$ on the release by human alveolar macrophages of factors controlling fibroblast functions. Biochem. Biophys. Res. Commun. 129,560, 1985.

25. Rola-Pleszczynski, M., and Lemaire, I. Leukotrienes augment interleukin-1 production by human monocytes. J. Immunol. 135,3958, 1985.

26. Rouzer, C.A., Scott, W.A. Cohn, Z.A., Blackburn, P., and Manning, J.M. Mouse peritoneal macrophages release leukotriene $\mathbf{C}$ in response to a phagocytic stimulus. Proc. Natl. Acad. Sci. U.S.A. 77, 4928, 1980.

27. Schmidt, J.A., Mizel, S.B., Cohn, D., and Green, I. Interleukin1, a potential regulator of fibroblast proliferation. J. Immunol. $128,2177,1982$.

28. Snyder, D.W., and Krell, R.D. Pharmacological evidence for a distinct leukotriene $\mathrm{C}_{4}$ receptor in guinea pig trachea. J. Pharmacol. Exp. Ther. 231,616, 1984.

29. Tsukamoto, Y., Helsel, W.E., and Wahl, S.M. Macrophage production of fibronectin, a chemoattractant for fibroblasts. J. Immunol. 127,673, 1981.

30. Wahl, S.M., and Wahl, L.M. Modulation of fibroblast growth and function by monokines and lymphokines. Lymphokines. 2,179, 1981.

31. Wahl, S.M., and Wahl, L.M. Regulation of macrophage collagenase, prostaglanin, and fibroblast-activating-factor production by antiinflammatory agents: Different regulatory mechanisms for tissue injury and repair. Cell. Immunol. 92,302, 1985.

32. Wharton W., Gillespie, G.Y., Russell, S.W., and Pledger, W.J. Mitogenic activity elaborated by macrophage-like cell lines acts as competence factors for BALB/c 3T3 cells. J. Cell. Physiol. 110,93, 1982.

33. White, J.R., Naccache, P.H., Molski, T.F.P., Borgeat, P., and Sha'afi, R.I. Direct demonstration of increased intracellular concentration of free calcium in rabbit and human neutrophils following stimulation by chemotactic factor. Biochem. Biophys. Res. Commun. 113,44, 1983. 